\title{
The Effectiveness of Using Mobile Learning Methods in Geometry for Students with Different Initial Mathematical Performance
}

\author{
Panagiota Panteli, Areti Panaoura* \\ Frederick University Cyprus \\ E-mail: pre.pm@frederick.ac.cy
}

\begin{abstract}
The present study investigated the use of mobile learning on students' (with different initial mathematical performance), understanding in geometry. The sample of the study was consisted of $1625^{\text {th }}$ grade students at secondary education (16-17 years old) who were divided into experimental and control group. For the mobile learning the Euclidea app was installed by the experimental group in their smart phones and it was used at the teaching of specific geometrical concepts. Emphasis was given on students' experiences. Although there were not any statistically significant differences between the two main groups concerning their performance, further analyses of the characteristics of the experimental group indicated that the mobile learning method was more efficient for students with low or medium performance than those with high initial performance on mathematics, The results highlight that there is not any "ideal" method in the teaching of mathematics, as there is need for differentiation derived by inter-individual differences. Implications about the use of mobile learning in mathematics education are discussed, as the use of short term intervention programs cannot fulfil the goals of changing significantly the students' mathematical performance.
\end{abstract}

Keywords: mobile learning, geometry, secondary education, Euclidea app

\section{Introduction}

The National Council of the Teachers of Mathematics, an organisation with decisions which influences the stakeholders in mathematics education worldwide, many years ago through the publication of mathematical standards underlined the necessity to use technology as a tool which can accelerate the development of mathematical thinking, in order to take advantage of the significant role it already has on students' life (NCTM, 2000). Today we are not talking only about the technology oriented generation, but about the generation of students who are surrounded by digital technology since their born. Mobile phones are part of daily student lives, both inside and outside the classroom ${ }^{[1]}$ and consequently are part of their informal education. According to UNESCO by the end of 2012 the number of mobile devices was estimated to exceed the world's population ${ }^{[2]}$. Mobile technology has been recognized as one of the most important innovations that influenced teaching and learning ${ }^{[3]}$ and there is an increased research interest on the introduction and implementation of mobile learning at the context of formal education ${ }^{[4]}$.

Mobile learning processes have been used in authentic environment places such as museums ${ }^{[5]}$, zoo ${ }^{[6]}$ and parks ${ }^{[7]}$. Designing effective mobile learning interventions requires holistic understanding of how people use and view any type of technology ${ }^{[2]}$. Although the mobile devices have increasingly power and they transformed the ways people communicate and work with data of various forms, in the education context, mobiles are often seen as a threat to the "serious" work of school ${ }^{[8]}$.

Although in Cyprus the New Curriculum in Mathematics (2015) highlights the necessity to use technology in teaching, there is still a discussion about the availability of wifi at school, the rules about the use of mobile phones at school and other similar concerns which do not allow mobile learning methods to be used in the expected level. Undoubtedly the adaptation of mobile learning presupposes the development of appropriate planning for the use of teaching processes for the development of relevant learning strategies ${ }^{[9]}$.

The present study examines the use of mobile devices as teaching and learning tools at the mathematics education of Cyprus, a country with a centralized educational system which has recently developed new Curriculum at many different subjects, as a part of an attempt for educational reform. It emphasizes at the same time the use of inquiry-based teaching 
processes which activate students' curiosity, creativity and problem solving skills. Especially in the case of geometry, the use of technology is proposed in order to facilitate the examination or investigation of mathematical concepts. For example it is easier to examine the relation of the radius and the area of a circle by using technological tools of dynamic geometry (such as Euclidraw or Sketchpad) rather than the traditional teaching tools. At the same time the use of technology leads to direct feedback which activates students' self-regulatory strategies in the case of a mistake ${ }^{[10]}$, while traditional methods lack immediate feedback and reduce students' interest in learning ${ }^{[11]}$.

The aim of the present study was to examine the effectiveness of using the mobile learning in the teaching of mathematics at secondary education. The aim concentrated on students' perspective during the implementation of the intervention program and the possible diversity of the effectiveness based on their initial differences on mathematical performance. Many times the same interventions are used for all the students, although the curriculum underlines the need for diversity and the fact that there are inter-individual differences based on personality, cognitive performance and previous experiences. We believe that the inter-individual differences may have a predominant role on the accountability of any intervention. For example the initial students' ability of information processing contributes significantly on their mathematical performance and their metacognitive behaviour during problem solving ${ }^{[12]}$. In the case of the secondary education we believe that students' different mathematical performance has to be examined further as a factor of differentiation concerning the effectiveness of using the mobile learning as a teaching tool for understanding the mathematical concepts. The posed research question which is examined at the present paper is a part of a project on students' beliefs, self-efficacy beliefs, motivations, skills and knowledge on using the mobile learning as tools for understanding the geometrical concepts.

\section{Theoretical framework}

Mobile learning was difficult to be defined at the beginning of the new century. It was posed as something new which was difficult to be conceptualized and discussed ${ }^{[13]}$. It is "any educational provision where the sole or dominant technologies are handheld or palmtop devices" (p. 262). Mobile learning is the admixture of distance learning method and electronic learning or it is the next generation of e-leaning ${ }^{[14]}$. The use of portable equipment such as mobile phones, ipads, personal digital assistants and tablet makes the mobile learning having no place and time limitations. The availability of the mobile technologies is probably the strongest argument for its use. It is unique that it can be accommodate both formal and informal learning in collaborative or individual learning modes, and within almost any context ${ }^{[15]}$.

\subsection{Mobile learning in education}

There are studies about the use of mobile technology at different educational levels, at different courses and based on the teaching or the learning perspective. For example ${ }^{[16]}$ examined the religious teachers' readiness to use mobile phones as teaching tools, while ${ }^{[17]}$ examined the use of mobile learning in the teaching of Biology. There are a rising number of conferences about mobile learning and relevant organizations foresee that mobile technology will soon become common place in both formal and informal education ${ }^{[2]}$.

With the increase of the availability of mobile devices there is a concomitant rise in the level of interest on examining the purpose and extent of mobile learning in education. The majority of the studies about the use of mobile phones took place in formal education, and mainly in the field of science education ${ }^{[18]}$. In the case of Greece, Kalogiannakis and Papadakis developed a teaching intervention in the context of an environmental education program ${ }^{[19]}$. In the case of mathematics education there are references derived by generalized conferences on mobile learning and by conferences on the use of technology in education. At the same time most studies concentrated on primary education and higher education in the case of prospective teachers. It seems that secondary school teachers are less likely than elementary school teachers to ask their students to use technology frequently, and when they do it they prefer to be in language courses and social studies ${ }^{[20]}$

Researchers found several problems concerning mobile teaching and learning such as (a) the change of traditional teaching curriculum and materials into digital contents, (b) the design of appropriate mobile devices and teaching methods, (c) the development of interactive functions on mobile programs and the teachers' education or training on using mobile teaching methods ${ }^{[3]}$. Similar problems or difficulties were found in higher education ${ }^{[21]}$, as instructors feel more confident to use the face-to-face teaching which has been traditionally used as relevant for many decades. In secondary education more practical difficulties concerning the implementation of mobile learning have been identified such as the lack of resources due to financial limitations, the lack of effective educational policy in order to have the relevant permission to use it; the lack of skilled instructors for effective implementation and the reservations of parents due to the perceptions about 
the use of mobile devices ${ }^{[22]}$. We believe that the difficulties and teaching obstacles which are identified about a subject or domain are in most cases similar at other domains as well. It seems that the investigation of technology in education, either by traditional means or by mobile devices, face obstacles that lies mainly on teachers' knowledge, skills and experiences ${ }^{[23]}$.

\subsection{The teaching and learning framework for the use of mobile learning in education}

Kearney and Maher's review indicated that research studies have attempted to examine mobile learning through identified theoretical perspectives and frameworks such as activity based approaches, authentic learning, action learning and experiential learning ${ }^{[24]}$. However Crompton et al. indicated that $40 \%$ of the time researchers designed mobile learning activities which are characterized by the behaviorist approach to learning ${ }^{[18]}$. Mobile technologies are supposed to support constructivist learning through active learning activities ${ }^{[25]}$. Although many studies have found that mobile teaching has positive impact on learning, the developed mobile learning programs are very "different in terms of course design, target group, learning methods and implementation environment" (p. 22) ${ }^{[3]}$.

Even in the case that both pre-service and in-service teachers have a positive belief in integrating mobile teaching tools in order to promote student learning, it is important how they integrate them ${ }^{[20]}$, whether there are related with collaborative learning and the emphasis is on the concept of a community of learners ${ }^{[26]}$. Teachers need to be educated on the benefits of incorporating mobile technology into their classrooms and then trained on how to integrate it effectively ${ }^{\text {[27] }}$. Mobile learning is often approached as an innovative method to teach, but quite often without the proper understanding of the implications on the pedagogy of the learning process in this setting ${ }^{[28]}$. Therefore mobile technology has ascended as a top priority of training programs around the world ${ }^{[15]}$. It is become an urgent matter to propose pedagogical approaches appropriate in respect to the complex educational dynamics ${ }^{[29]}$.

Mobile devices can be effective educational platform, due to the fact that mobile devices are easily accessible and help in making the students' learning more interesting, interactive, widely available and flexible ${ }^{[14]}$. They list a number of benefits of using the mobile learning: anytime access to content, anywhere access to content, enhance student-centered learning, support differentiation of student learning needs and personalized learning, and enhance interaction between and among the students and instructors. They underline the necessity to use the mobile technologies for self-evaluation and reflection throughout the learning process.

Fabian, et al. investigated the effects of using mobile technologies to students' attitudes and achievement ${ }^{[25]}$. Their results indicated that the use of mobile technologies elicit positive responses from students both in terms of how they perceive the mobile activities and how it improved their performance. Taleb, et al. highlighted the opportunity for diversity of training methods of mathematics and indicated that there is a positive and significant relation between using mobile learning and students' participation in mathematics ${ }^{[27]}$. Mobile devices allow students to explore their environment and in the case of "trigonometry, measurement and geometry there can be used to analyze the notion of a moving object that appears in a digital video" (p. 575) ${ }^{[30]}$. It seems that mobile device use increases the learners' tendency to engage in selfdirected learning and it stimulates curiosity beyond the classrooms ${ }^{[15]}$.

\subsection{The use of mobile learning in mathematics education}

We cannot find yet too many studies about the effectiveness of using mathematical applications at the different educational levels. The review of Soykan and Uzunboylu indicated that only $2 \%$ of the studies were about the use of mobile learning in mathematics ${ }^{[31]}$. Few of them are in primary education ${ }^{[32]}$ and most of them in secondary education ${ }^{[25]}$. Most of them had positive results [33] and few of them neutral effects ${ }^{[34]}$. Ariyanto, et al. ${ }^{[35]}$ examined the role of the use of mobile phone applications in mathematics learning. Students' learning results indicated significant improvement and students showed an interest in using the specific application. In the case of geometry, Chang et al. (2016) suggest the use of mobile learning for geometrical figure apprehension ${ }^{[36]}$, while Supandi et al. ${ }^{[1]}$ suggest if for the understanding of trigonometry. One of the major presuppositions for the use of mobile learning in the teaching of mathematics is its acceptance as a tool for investigation and exploration of mathematical concepts ${ }^{[37]}$.

The most usual applications which are used for the teaching of Geometry are Euclidraw, Cabri, Sketchpad and Geogebra (some of them were used mainly the previous years). Their major characteristic is the dynamic perspective of examining the geometrical concepts. For example students are able to understand easily the relationship between the radius and the diameter of a circle by transforming the circle size. The use of mobile applications facilitates the establishment of an interactive learning environment for the understanding of difficult geometrical concepts ${ }^{\text {[36] }}$.

Alkhateeb and Al-Duwairi propose the integration of mobile applications of Geogebra dnd Sketchpad at the teaching of geometry at the university level ${ }^{[38]}$. Their study showed that both applications have positive effects on students' achievements. Especially in the case of Geometry the MobileMath was created by Wijers et al. in 2008 in order to help students experience mathematical concepts in the physical world, and the MobiMath was created by Tanjney et al. in 
2010 in order to facilitate the teaching of difficult geometrical concepts ${ }^{[39]}$. The use MobiMath indicated that it enhanced collaborative learning experiences and communication between the members of the groups who had to solve a problem.

The use of Euclidea app

Euclidea is the application that was used in the present study. It was developed by the Russian Horis International Limited, which specializes in apps with a strong math background. The main emphasis of the specific application is to solve geometric problems by using a compass and straightedge constructions. Examples of such problems are to bisect a given angle or construct the perpendicular bisector to a segment. The main advantage of the application is that it is free and it can be easily installed at smart phones.

The application offers direct feedback and it is characterized by many strengths. It highlights in yellow a construction, to indicate that it is correct and shows a reward pop-up message. Its use is extremely easy and friendly, as at the initial stage the user has to use only two tools, a compass and a straightedge. Some of the constructions you have to make are particularly significant, and after you make them they are added as shortcuts to the toolbox. The additional tools allow you to make common operations without explicitly doing all the steps. An important and useful feature is that the constructions are inherently dynamic. By selecting an arbitrary point of your construction and move your finger on the display, the construction reshapes, helping you to verify that it works in general.

This application includes various levels and each one is consisted of certain tasks. There is an increase at the level of task's difficulty in relation to the increase of the level. Solving the task of one level, the app automatically "unlocks" the next one, based on the specific hierarchical structure.

\section{Methodology}

\subsection{Experiment design}

To evaluate the effectiveness of mobile learning in the teaching of geometry at secondary education, an experiment was conducted and consequently pre and post tests were used for the comparison. The purpose of the experiment was to compare the students' of the experimental group learning achievements, after their participation in learning activities based on the use of a smart phone app called Euclidea, with the respective achievements of the control group (traditional teaching approach was followed based on the guidelines of the curriculum)

\subsection{Participants}

The sample was consisted of 162 Cypriot high school students from 8 classes of 2 lyceums. They were at the $5^{\text {th }}$ grade at secondary education (mean age of 16 years old) and they attended extra hours on Maths, as it was selected by them as one of their major courses. An equal number of students (81) constituted the two groups: the experimental group (EG) and the control group (CG). Actually their classes were posed randomly as part of the experimental or the control group.

Six math teachers taught mathematics at the 8 classes during the implementation of the intervention. Three teachers taught the specific geometrical concepts at the Control group (CG) and they used the traditional teaching approach according to the guidelines and processes of the National Curriculum. The other three teachers used the Euclidea app in order to teach the same geometrical concepts at the experimental group of students. The last group of teachers attended previously a training offered by the researchers. During the experiment their teaching processes and practices were videotaped in order to ensure the validity of their practices in relation to the given guidelines. The sample of the teachers and the respective sample of students were not representative as researchers based on teachers' voluntary contribution due to teachers' difficulties with the relation of time allocation and the quantity of the concepts they have to teach.

\subsection{Research process}

The schedule and the whole research process which was followed are presented at Figure 1. Firstly during the first 45-minute meeting, the pre-test was used in order to measure the participants' van Hiele level of geometrical thinking.

Then, an intervention programme was developed and implemented by using the mobile learning for the experimental group, about the teaching of the geometrical concepts which are presented at the school textbook. In the case of Cyprus, there is a common Curriculum, common textbooks for all the students and teacher guide with the expected teaching processes and learning outcomes. The CG class students were taught based on the traditional teaching approach which is presented at the teacher guide, according to which for the geometric constructions, both the teacher and the students used geometric instruments (a ruler and a compass). Teaching the same concepts in the EG, their teachers asked them to use the Euclidea app which they had already installed on their smart phone. Both groups performed the same activities as well as finding the solution of the same problems from the school textbook in order to examine the impact of the different teaching methods. During, the sixth and final 45-minute session all students from both groups (CG and EG) were given a common 
test relevant to the covered mathematical content.

Pictures 1 and 2 present the work of the experimental group during the intervention. Students after getting acquainted with the application tools for a few minutes, their teacher presented them on the board how they could investigate a statement and construct a relevant proof. The teacher had in their disposal a smart device-in this case an iPad- which was
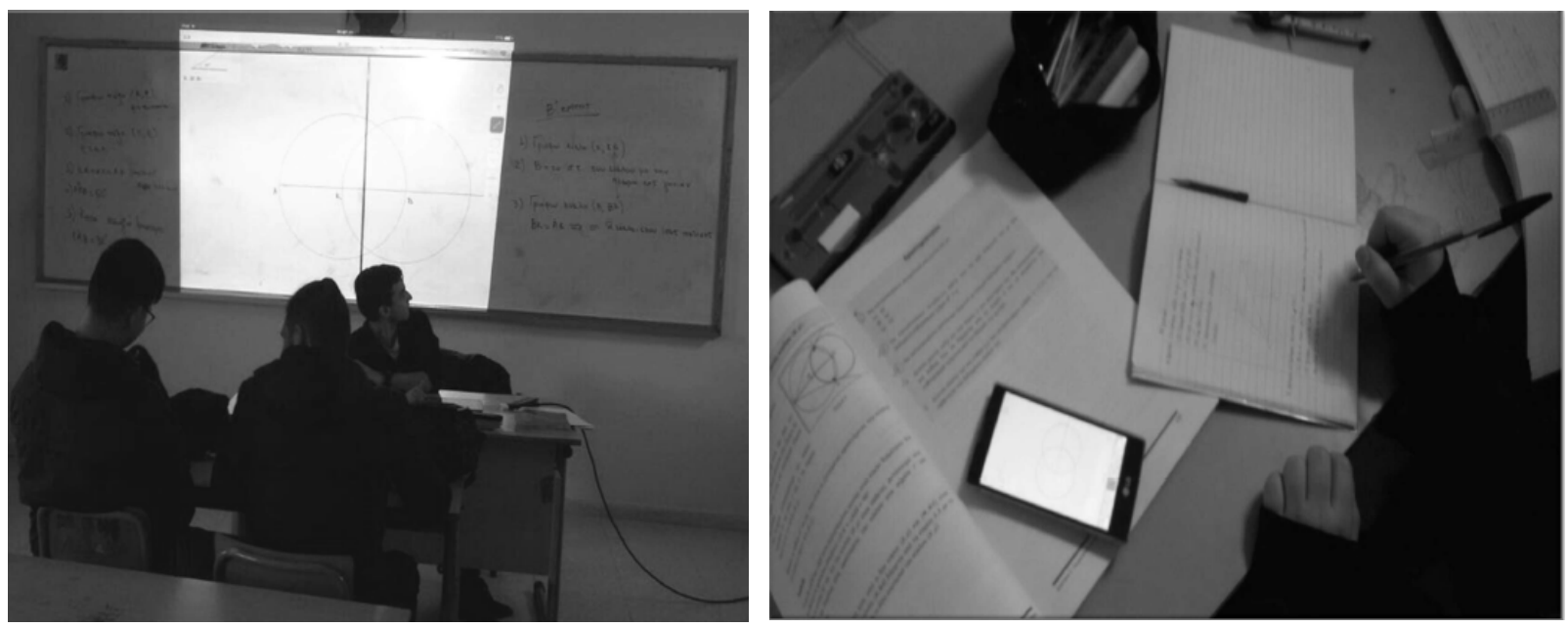

connected to a video projector. Students had the opportunity to dynamically reshape the construction in order to verify that the construction can work under any circumstances. They were able to continue their work whenever they wanted and they could work on the following up stages if they had the time and the respective wish or will to do it.

Picture 1. Teaching processes for the control group

Picture 2. Learning Activities of the experimental group

After the experience of the intervention program, the participants of the experimental group completed a questionnaire, in order to examine their conceptions and impressions derived from the use of the mobile learning devices at the learning process. Finally, personal semi-structured interviews were conducted by a number of EG students, targeting in the confirmation of the questionnaire's results concerning their "Views and Impressions" as well as the valid interpretation of these results. The research process, which was used, is presented briefly at Figure 1. The present study is a part of a project which examined students' performance, motivation, collaboration during the activities and teachers' teaching behaviour. At the present paper we examine only the aspects which are related with the previously presented research aim.

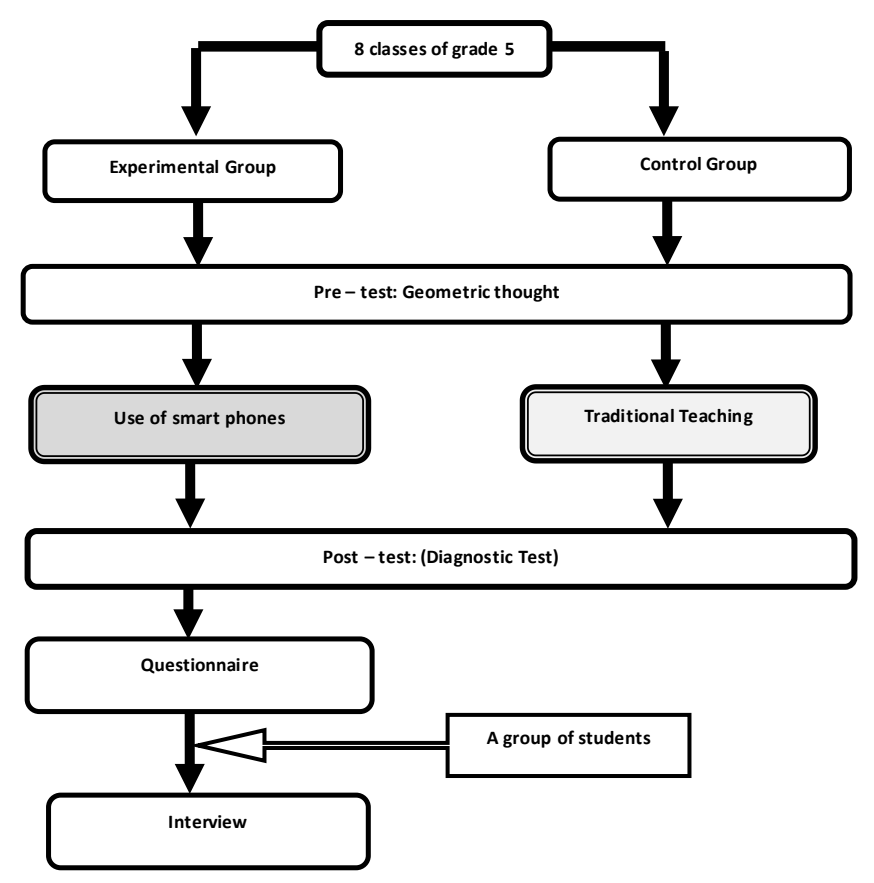

Figure 1. The research process 


\subsection{Research instruments}

Geometrical thinking thought Pre-test: The VHGT (van Hiele Geometry Test) was used as pre-test in order to identify students' van Hiele levels. It consisted of 25 multiple choice tasks. Questions 1 through 5 represent Level 1, questions 6 through 10 represent Level 2, and so on. The correct correspondence at least three tasks of each level was a sufficient indication that the student achieved to have the characteristics of the specific level. The construction of the test by van Hiele, was based on the assumption that by this procedure each student can be enlisted in the highest level according to his/ her abilities without being able to skip any hierarchical level of the geometrical thinking ability.

Geometric performance post-test: Researchers constructed a test relevant to the topics covered during the teaching of the unit. The content validity of the test was examined by the 6 maths teachers who took part at the research. Then the appropriate correction guide was constructed so that all teachers would be able to grade the tests based on the same criteria. The lowest grade could be 1 and the highest could be 20 .

Post questionnaire on the students' conceptions about the experience: It was answered only by the EG students after completing the intervention. The questionnaire was consisted of 15 five-point Likert scale items (1="strongly disagree", $5=$ "strongly agree"). Items were adapted from two different questionnaires that were used in the past for similar purposes. The first questionnaire was constructed by Hwang et al. ${ }^{[9]}$ and the second one by Chu et al. ${ }^{[6]}$.

\section{Results}

\subsection{The effect of the intervention on students' performance}

To evaluate the effectiveness of using the mobile devices for the teaching of a unit in geometry we have examined the differences on the students' performance between the experimental and the control group. Firstly, a descriptive statistical analysis was conducted for the examination of the respective means and standard deviations (Table 1). As it was expected the means for the EG during the post-test was higher than the respective mean of the CG. To be more specific, the lowlevel students on geometrical thinking based on van Hiele's model (1 and 2) of the experimental group presented a higher mean than the respective students of the control group. The results were similar in the case of the students of the medium level (EG: . Only in the case of the students of the high level the performance of the experimental group ( was slightly lower in relation to the equivalent performance of the control group .

In other words, it is clear that the experimental process helped the lower and medium level students to acquire a higher mean compared to the equivalent students of the control group. On the other hand, the already high-level students had minimum improvement changes compared to the respective since they were already at a high level, with possibly minimum chances for improvement.

Table 1. Students' of control and experimental group performance at the post test in respect to their initial levels of geometrical thinking

\begin{tabular}{ccccc}
\hline & \multicolumn{4}{c}{ Geometrical performance } \\
\hline $\begin{array}{c}\text { Levels of geometrical } \\
\text { thinking }\end{array}$ & M.O. & T.A. & M.O & T.A \\
Low & 4.87 & 2.66 & 5.92 & 2.09 \\
Medium & 12.80 & 2.54 & 13.50 & 2.95 \\
High & 18.84 & 1.34 & 18.60 & 1.43 \\
Total & 11.09 & 6.02 & 12.23 & 5.45 \\
\hline
\end{tabular}

A two-way ANOVA was conducted in order to examine the effect of teaching group and van Hiele level of each one, on students' performance in geometry post-test. Simple main effects analysis showed that there were not any statistically significant differences between the two groups of students). As it was expected the classification of students based on the van Hiele's level of geometrical thinking seemed to have significant main effect. Their initial geometrical differences remained after the short term intervention indicating that short term interventions cannot permit us to face the students' inter-individual differences based on their initial performance.

Finally, there was not any statistically significant interaction between the effects of teaching group and van Hiele's level of geometrical thinking on students' performance. There is a tendency to expect statistically significant changes after an intervention program and probably statistically significant differences between the experimental and the control group. In the specific case it is important to identify that a short term intervention can have positive effects on few students or few specific dimensions, however it is not enough in order to achieve significant effects. 


\subsection{Students' conceptions and experiences}

To investigate the students' conceptions after the experiences of the implementation of the intervention program, the analysis of the questionnaire and the analysis of the interviews were conducted. Results of the descriptive analysis of the statements of the questionnaire are presented in Table 2. It seems that the vast majority of students seem to have positive views on both this application as well as the teaching method of using mobile learning at the teaching of mathematics in general. Specifically all the questions (5) related to the application presented mean $\geq 3.5$. More specifically, $72 \%$ of the students agreed or strongly agreed with the statement that the application was used easily, while almost $70 \%$ of the students mentioned that it did not take them much time to learn how to use it. Additionally, an almost equal percentage of students found the functions of the app quite useful for the achievement of their learning goals.

During the personal interviews, a girl mentioned how much she liked being able to test various possible solutions, fast and without much effort, without needing to erase and rewrite the shapes again and again. A boy said that he liked being able to create circles so easily, as he found it rather difficult and boring to keep using the compass.

As far as the feedback provided by the use of the app, a student mentioned that it was really important that they had a direct feedback on their construction, as the colour turned to yellow. However a different student disagreed and he said that the shape turning yellow wasn't particularly helpful for him, since he would rather get a message that would give him some clue on how to make it right. He wanted a qualitative type of individual feedback in order to know how to react.

Based on the results presented at Table 2 it seems that the students' conceptions about using the specific learning method were actually quite positive since 6 out of 7 questions related to the specific topic appear to have mean $\geq 4$. It is worth mentioning that $80 \%$ of the students either agreed or strongly agreed with the statement that the use of smartphones has made the learning activities by far more interesting, while the same percentage said that they would prefer to apply this approach to other subjects as well. Additionally, $75 \%$ of the students either agreed or strongly agreed with the statement that learning through the use of a smartphone is far more interesting and it is, at the same time, a lot more challenging compared to traditional teaching.

Table 2. Students' conceptions about the use of mobile learning after the experience

\begin{tabular}{|c|c|c|c|c|c|c|}
\hline \multirow[b]{2}{*}{$\mathbf{A}$} & \multirow[b]{2}{*}{ Application } & \multirow[b]{2}{*}{$\mathrm{M}$} & \multirow[b]{2}{*}{ S.D } & \multicolumn{3}{|c|}{ Views / Impressions } \\
\hline & & & & Negative & Neutral & Positive \\
\hline 1 & The application can easily be used. & 4.17 & 0.84 & $0 \%$ & $27.8 \%$ & $72.2 \%$ \\
\hline 2 & $\begin{array}{l}\text { It only took me a short time to fully understand how to use the } \\
\text { application. }\end{array}$ & 4.11 & 0.92 & $2.8 \%$ & $27.8 \%$ & $69.4 \%$ \\
\hline 3 & $\begin{array}{l}\text { Using the feedback provided by the application enabled me to concentrate } \\
\text { on the processes. }\end{array}$ & 3.86 & 0.87 & $5.6 \%$ & $27.8 \%$ & $66.7 \%$ \\
\hline 4 & The functions provided by the application were useful. & 3.83 & 0.91 & $5.6 \%$ & $25.0 \%$ & $69.4 \%$ \\
\hline 5 & $\begin{array}{l}\text { The feedback provided by the application was useful to face few of my } \\
\text { misconceptions. }\end{array}$ & 3.61 & 0.93 & $8.3 \%$ & $44.4 \%$ & $47.2 \%$ \\
\hline B. & \multicolumn{6}{|c|}{ Teaching approach } \\
\hline 6 & I like to learn new uses of my smart phone. & 4.11 & 0.98 & $5.6 \%$ & $16.7 \%$ & $77.8 \%$ \\
\hline 7 & I wish this innovative approach could be applied to other courses as well. & 4.17 & 0.97 & $5.6 \%$ & $13.9 \%$ & $80.5 \%$ \\
\hline 8 & $\begin{array}{l}\text { I would like to acquire new knowledge by using my smart phone in the } \\
\text { future. }\end{array}$ & 4.17 & 1.03 & $5.6 \%$ & $19.4 \%$ & $75.0 \%$ \\
\hline 9 & The use of smart phone makes learning activities more interesting. & 4.14 & 1.02 & $8.4 \%$ & $11.1 \%$ & $80.5 \%$ \\
\hline 10 & This way of learning is interesting and challenging. & 4.08 & 1.13 & $8.4 \%$ & $16.7 \%$ & $75.0 \%$ \\
\hline 11 & During the learning activities I engaged myself in the learning tasks. & 3.64 & 1.10 & $13.9 \%$ & $25.0 \%$ & $61.1 \%$ \\
\hline 12 & I would recommend the use of this learning approach in peers. & 4.00 & 0.92 & $5.6 \%$ & $16.7 \%$ & $77.8 \%$ \\
\hline
\end{tabular}

It is also noteworthy, that the students who had come to the first lesson without having installed the app on their phones in advance, mainly due to lack of space on their device, after seeing their classmates using it, they decided to delete other applications or data from their phones in order to install that particular app. The teachers were also impressed by the fact that students who had previously felt bored and without any motivations in the lesson, they were next showing a lot more interest and wanted to be more involved in the learning activities.

The majority of students clearly showed their enthusiasm of the processes during the personal interviews. More specifically, a student said that it was unbelievable when the teacher asked them to take up their phones to do the lesson, as he had never thought that the day would come when they would be using their smartphones in class. Another student 
admitted that he had been feeling bored during the maths lesson lately, but the teacher had made that particular lesson a lot more interesting due to the use of app. He also stated that from now on, he would be paying more attention in class and study harder at home. Finally, there was even one pupil who said that his friends from other classes would definitely feel jealous, once they were told how the new lesson was conducted.

On the other hand, some negative feelings or conceptions had been expressed by certain students, but this can be attributed to the fact that they had not been adequately prepared or ready to work by this different way. More specifically, a girl stated that she had felt very stressed and wished they would not be tested on the specific unit. She continued by saying that they did not have proper lessons the other days; instead they would just play on their phones. This shows us, that there is a group of students who have been so attached to the traditional teaching approach that anything beyond this is not considered an "actual lesson". The most impressive result which has to be examined further was that students who had those types of conceptions, they had at the same time high performance in mathematics.

\section{Discussion}

Undoubtedly mobile learning methods based on the advantage of the flexible access at any time and place ${ }^{[40]}$. It is unique in that "it can accommodate both formal and informal learning in collaborative or individual learning modes and within almost any context" ${ }^{[3]}$. When we suggest the use of technology in education we have to be aware of the continuous technological change that is taking place ${ }^{[41]}$. The present study aimed to examine the role of the use of mobile learning in students' understanding of geometrical concepts and the impact of short term teaching method of using it as teaching tool.

The results of the present study indicate that mobile learning has a positive effect on motivating students towards mathematics. However it seems that results are not the same for all the students. Students with high performance in mathematics indicated that they had a stronger resistance in reacting positively in different teaching methods. This is mainly due to the fact that those students had already developed learning strategies which were successful for them and they were not able to understand the reasons for trying something different ${ }^{[29]}$. Those students have to try a new method for longer duration and relate its probable positive effects with the already developed strategies based on their learning style.

Students with higher initial performance based on the van Hiele's geometrical levels had the highest performance after the intervention. The inter-individual differences remained the same. It was important the indication of a tendency for an increase on students' of the medium and low level performance on geometry, although the difference was not statistically significant.. It would be naïve to believe that such a short-term intervention could change dramatically the students' initial differences and actually this was not the aim of the study. The differences based on their initial performance could indicate an indirect relation, while the direct relation could be based on their self-efficacy beliefs on solving mathematical tasks. It seems that results would be probably better and more stable in the case of using similar methods for a longer time and probably in different contexts. Students' positive experiences are important and hopeful messages for enforcing the following up steps. The use of mobile technology provides education with the opportunity to reimagine teaching and learning processes and framework ${ }^{[26]}$. In-service teachers' training programs have to be developed and implemented in order to face initial concerns and worries and in order to develop their skills, knowledge and abilities to face the teaching obstacles ${ }^{[23]}$.

The present study concentrates on the specific domain of geometry, however it is obvious the tendency to incorporate the mobile learning methods for the exploration, investigation and understanding of many different mathematical concepts. We have always to keep in mind that it is a teaching tool in order to facilitate the understanding and the construction of the concepts. It cannot be used without a clear pedagogical framework and it cannot be suggested as a method appropriate for all the students. We are not trying to find any ideal methods. There are always inter-individual differences which lead to a necessity of diversity. A future study could concentrate on the evaluation of the use of mobile learning methods for students with different cognitive styles, learning styles and initial conceptions and efficacy beliefs about the use of technological tools as facilitators of the learning process. At the same time a future study has to overcome the administrative obstacles of the centralized educational system and implement an intervention program of using the mobile teaching methods in geometry for a longer time. Finally a future study could examine teachers' knowledge, skills, beliefs, self-efficacy beliefs and practices on using the mobile learning as teaching tool at secondary education in order to fulfil the goals of geometry education in particular and mathematics education in general. 


\section{References}

[1] Supandi S, Ariyanto L, Kusumaningsih W, Aini A. Mobile phone application for mathematics learning. In Journal of Physics: Conference Series. 2018; 983(1): 1-5.

[2] UNESCO . The future of mobile learning. Implications for policy makers and planners. 2013; France.

[3] Zhang M, Trussell R, Gallegos B, Asam R. Using math apps for improving student learning: An exploratory study in an inclusive fourth grade classroom. TechTrends. 2015; 59(2): 32-39.

[4] Bidin S, Ziden A. Adoption and Application of Mobile Learning in the Education Industry. Procedia-Social and Behavioral Sciences. 2013; 90: 720-729.

[5] Sung Y, Hou H, Liu C, Chang K. Mobile guide system using problem-solving strategy for museum learning: a sequential learning behavioural pattern analysis. Journal of computer assisted learning. 2010; 26(2):106-115.

[6] Chu H, Hwang J, Tsai C, Tseng J. A two-tier test approach to developing location-aware mobile learning systems for natural science courses. Computers \& Education. 2010; 55(4): 1618-1627.

[7] Lai C, Yang J, Chen F, Ho C, Chan T. Affordances of mobile technologies for experiential learning: the interplay of technology and pedagogical practices. Journal of Computer Assisted Learning. 2007; 23(4): 326-337.

[8] White T, Martin L. Mathematics and Mobile Learning. Tech Trends. 2014; 58 (1): 1-12.

[9] Hwang G., Shi Y, Chu H. A concept map approach to developing collaborative Mindtools for context-aware ubiquitous learning. British Journal of Educational Technology. 2011; 42(5): 778-789.

[10] Panaoura A. Self-regulatory strategies during problem solving by using an inquiry-based approach: "Make sense of problems and persevere in solving them". In: Newton L. (eds.) Problem-Solving: Strategies, Challenges and Outcomes: Nova Science Publishers; 2016, p.223-237.

[11] Papadakis S, Kalogiannakis M, Zaranis N. The effectiveness of computer and tablet assisted intervention in early childhood students' understanding of numbers. An empirical study conducted in Greece. Education and Information Technologies. 2018; 23(5): 1849-1871.

[12] Panaoura A, Gagatsis A, Demetriou A. An intervention to the metacognitive performance self-regulation in mathematics and mathematical modeling. Acta Didáctica Universitatis Comenianae Mathematics. 2009; 9 : 63-72.

[13] Traxler J. Defining mobile learning. IADIS International Conference Mobile Learning. 2005: 261-266.

[14] Sarrab M, Elgamel L, Aldabbas H. Mobile learning (M-Learning) and Educational Environments. International Journal of Distributed and Parallel Systems. 2012; 3 (4): 31-38.

[15] Hagg J, Berking P. Design Considerations for Mobile Learning. In: Zhang Y. (ed.) Handbook of Mobile Teaching and Learning . Springer; 2015, p. 41-60.

[16] Nawi A, Hamzah M, Rahim A. Teachers acceptance of mobile learning for teaching and learning in islamic educations. Turkish online Journal of Distance Education. 2015; 16 (1): 184-192.

[17] Gedik N, Hanci-Karademirci A, Kursun E, Cagiltay K. Key instructional design issues in a cellular phone-based mobile learning project. Computers \& Education. 2012; 58(4): 1149-1159.

[18] Crompton H, Burke D, Gregory K. The use of mobile learning in PK-12 education: A systematic review. Computers and Education. 2017; 110: 51-63.

[19] Kalogiannakis P, Papadakis S. Combining mobile technologies in environmental education: a Greek case study. International Journal Mobile Learning and Organisation. 2017; 11 (2): 108-130.

[20] McCulloch A, Hollebrands K, Lee H, Harrison T, Factors that influence secondary mathematics teachers' integration of technology in mathematics lessons. Computers \& Education. 2018; 123: 26-40.

[21] Turbill J. Transformation of Traditional Face-to-Face Teaching to Mobile Teaching and Learning: Pedagogical Perspectives. In Zhang Y. (ed.) Handbook of Mobile Teaching and Learning. Springer; 2015. p. 221-234.

[22] Bano M, Zowghi D, Kearney M, Schuck S, Aubusson P. Mobile learning for science and mathematics school education: A systematic review of empirical evidence. Computers and Education. 2018; 121: 30-58.

[23] Papadakis S. Evaluating pre-service teachers' acceptance of mobile devices with regards to their age and gender: a case study in Greece. International Journal of Mobile Learning and Organisation. 2018; 12(4): 336-352.

[24] Kearney M, Maher D. Mobile learning in maths teacher education: Using ipads to support pre-service teachers' professional development. Australian Educational Computing. 2013; 27 (3): 76-84.

[25] Fabian K, Topping K, Barron I. Using mobile technologies for mathematics: effects on student attitudes and achievement. Education Technology Research Development. 2018; 66: 1119-1139.

[26] Heflin H, Shewmaker J, Nguyen J. Impact of mobile technology on student attitudes, engagement and learning. Computers and Education, 2017; 107: 91-99.

[27] Taleb Z, Ahmadi A, Musavi M. (2015). The effect of m-learning on mathematics learning. Procedia - Social and Behavioral Sciences. 2015; 171: 83-89.

[28] Petrakieva L. (2015). Mobile Technologies and Learning: Expectations, myths and reality. In Zhang Y (ed.) Handbook 
of Mobile Teaching and Learning. Springer; 2015. p.973-982.

[29] Figueredo O, Villamizar J. (2015). Framework for Design of Mobile Learning Strategies. In Zhang Y. (ed.), Handbook of Mobile Teaching and Learning. Springer; 2015 p.75-90.

[30] Lee H, Kautz J. Designing Mathematical Tasks within Mobile Learning Environments. In Y. Zhang (ed.), Handbook of Mobile Teaching and Learning. Springer; 2015, p.573-600.

[31] Soykan E, Uzunboylu H. New trends on mobile learning area: The review of published articles on mobile learning in science direct database. World Journal on Educational Technology. 2015; 7(1): 31-41.

[32] Shin N, Sutherland L, Norris C, Soloway E. Effects of game technology on elementary student learning in mathematics. British Journal of Educational Technology. 2012; 43(4): 540-560.

[33] Franklin T, Peng, L. Mobile math: Math educators and students engage in mobile learning. Journal of Computing in Higher Education. 2008; 20(2): 69-80.

[34] Lan Y, Sung Y, Tan N, Lin C, Chang K. Mobile-device supported problem-based computational estimation instruction for elementary school students. Journal of Educational Technology \& Society. 2010; 13(3): 55-69.

[35] Ariyanto S, Kusumaningsih W, Aini A. Mobile phone application for mathematics learning. International Conference on Mathematics, Science and Education, Conference Senses. 2018; 98(3): 1-5.

[36] Chang K, Wu L, Lai S, Sung Y. (2016). Using mobile devices to enhance the interactive learning for spatial geometry. Interactive Learning Environments. 2016; 24(4): 916-934.

[37] Eliasson J, Pargman T, Nouri J, Spikol D, Ramberg R. Mobile devices as support rather than distraction for mobile learners: Evaluating guidelines for design. International Journal of Mobile and Blended Learning. 2011; 3: 1-15.

[38] Alkhateeb M, Al-Duwairi A. The effect of using mobile applications (Geogebra and Sketchpad) on the students' achievement. International Electronic Journal of Mathematics Education. 2019; 14 (3): 523-533.

[39] Drigas A, Pappas M. A review of mobile learning applications for Mathematics. 2015. Available online: http://dx.doi. org/10.3991/ijim.v9i3.4420

[40] Voogt J, Erstad O, Dede C, Mishra P. Challenges to learning and schooling in the digital networked world of the $21^{\text {st }}$ century. Journal of Computer Assisted Learning. 2013; 29: 403-413.

[41] Tharayil S, Bowen M, Prince M, Nguyen K, Shekhar P, Finelli C, Waters C. Strategies to mitigate student resistance to active learning. International Journal of STEM Education. 2018; 5 (7): 1-16. 